\title{
Persistent Transcriptional Alterations after Hepatitis C Virus Elimination in Cell Culture ${ }^{\dagger}$
}

\author{
Victoria Castro 1, Gema Calvo 1, Juan Carlos Oliveros 2, Sofia Pérez-Del-Pulgar 3,4, \\ Xavier Forns ${ }^{3,4,5}$ and Pablo Gastaminza $1,4, *$ \\ 1 Department of Cellular and Molecular Biology Centro Nacional de Biotecnología-Consejo Superior de \\ Investigaciones Científicas, 28049 Madrid, Spain; vcastro@cnb.csic.es (V.C.); gcalvo@cnb.csic.es (G.C.) \\ 2 Bioinformatics Core Facility Centro Nacional de Biotecnología-Consejo Superior de Investigaciones \\ Científicas, 28049 Madrid, Spain; oliveros@cnb.csic.es \\ 3 Liver Unit, Hospital Clínic, Institut d'Investigacions Biomèdiques August Pi i Sunyer (IDIBAPS), \\ 08036 Barcelona, Spain; SOFIAPP@clinic.cat (S.P.-D.-P.); xforns@clinic.cat (X.F.) \\ 4 Consorcio Centro de Investigación Biomédica en Red de Enfermedades Hepáticas y Digestivas \\ (CIBERehd), 28029 Madrid, Spain \\ 5 Universitat de Barcelona, 08007 Barcelona, Spain \\ * Correspondence: pgastaminza@cnb.csic.es \\ † Presented at Viruses 2020-Novel Concepts in Virology, Barcelona, Spain, 5-7 February 2020. \\ Published: 17 June 2020
}

\begin{abstract}
Chronic hepatitis $\mathrm{C}$ virus (HCV) infection causes liver inflammation and fibrosis, which can lead to development of cirrhosis and hepatocellular carcinoma (HCC). The recent approval of direct-acting antiviral (DAA) drug combinations has revolutionized antiviral therapy against HCV. These drugs enable virus eradication in virtually all treated patients regardless of their genotype and liver disease status. Based on clinical parameters, it has been proposed that elimination of infected cells by reactivated immune responses may be dispensable for virus eradication in contrast to previously used interferon-based therapies. It is thus formally possible that the patients, who are declared cured, do indeed carry formerly infected cells that display irreversible alterations due to prolonged chronic HCV infection. Although transcriptional profiles of biopsies from cured patients have been previously studied, it is difficult to determine the precise mechanisms by which permanent alterations are established in the context of a heterogeneous tissue, often in patients with an underlying liver disease. Thus, we used cell culture models of persistent HCV infection to determine if $\mathrm{HCV}$ infection causes permanent transcriptional alterations in host cells after virus eradication. In these models, HCV infection causes profound alterations of host cell transcriptome that aim at regaining cellular homeostasis in the context of intracellular membrane rearrangements, interference with homeostatic processes, and persistent stress conditions, and permit cell survival even though the virus has colonized the host cell. In this context, we asked the question of whether the original homeostasis and original transcriptomic profile are regained in formerly infected cells after DAA treatment-mediated virus eradication. Our results indicate that a minor subset of transcriptional alterations persists even after virus eradication, suggesting that DAA-mediated eradication does not ensure the normalization of formerly infected cell homeostasis. Combined analysis of the transcriptional profiles in proliferating and growth-arrested cells suggests that several mechanisms underlie the establishment of permanent alterations.
\end{abstract}

Keywords: hepatitis C virus; direct-acting antiviral; transcriptional alterations

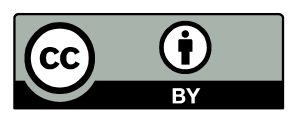

(C) 2020 by the authors. Licensee MDPI, Basel, Switzerland. This article is an open access article distributed under the terms and conditions of the Creative Commons Attribution (CC BY) license (http://creativecommons.org/licenses/by/4.0/). 